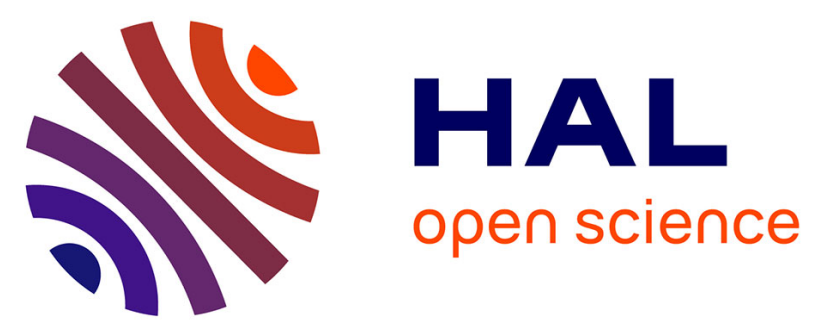

\title{
Software corrected hot wire thermal lag for the constant voltage anemometer featuring a constant bandwidth at the selected compensation setting
} Garimella R. Sarma, Geneviève Comte-Bellot, Thierry M. Faure

\section{- To cite this version:}

Garimella R. Sarma, Geneviève Comte-Bellot, Thierry M. Faure. Software corrected hot wire thermal lag for the constant voltage anemometer featuring a constant bandwidth at the selected compensation setting. Review of Scientific Instruments, 1998, 69 (9), pp.3223-3231. 10.1063/1.1149087 . hal01092018

\section{HAL Id: hal-01092018 https://hal.science/hal-01092018}

Submitted on 8 Dec 2014

HAL is a multi-disciplinary open access archive for the deposit and dissemination of scientific research documents, whether they are published or not. The documents may come from teaching and research institutions in France or abroad, or from public or private research centers.
L'archive ouverte pluridisciplinaire HAL, est destinée au dépôt et à la diffusion de documents scientifiques de niveau recherche, publiés ou non, émanant des établissements d'enseignement et de recherche français ou étrangers, des laboratoires publics ou privés. 


\title{
Software corrected hot wire thermal lag for the constant voltage anemometer featuring a constant bandwidth at the selected compensation setting
}

\author{
Garimella R. Sarmaa) \\ Tao Systems, Inc. Williamsburg, Virginia 23185 \\ Genevieve Comte-Bellot ${ }^{b)}$ \\ LMFA, Ecole Centrale Lyon, Ecully Cedex, France \\ Thierry M. Faure ${ }^{\mathrm{c})}$ \\ LEMFI, Universite P. et M. Curie, Paris 6, Orsay Cedex, France
}

(Received 2 June 1998; accepted for publication 29 June 1998)

\begin{abstract}
Software compensation. correction for thermal lag of a hot wire in the application of a constant voltage anemometer (CVA) for turbulence measurements in the boundary layer of a supersonic wind tunnel has been demonstrated. The CVA was used with a fixed compensation setting while measuring the in situ thermal lag (time constant) of the hot wire. Using the measured time constant, corrections are applied to the fixed compensation output of the CVA in postprocessing of the data. To demonstrate the flexibility of the approach it was used for two compensation settings at a test point to obtain the same results from both settings. A unique advantage of this approach is shown to be that for a given compensation setting in the CVA the bandwidth of the measurements for the test remains constant for all of the different test conditions and yields higher productivity. The results of the turbulence levels measured with this method agree with earlier research using other anemometers. Spectral plots of the mass flux and temperature and the measured in situ time constant responses under different conditions of the hot wire have been presented. (C) 1998 American Institute of Physics. [S0034-6748(98)05209-5]
\end{abstract}

\section{INTRODUCTION}

The constant temperature anemometer (CTA) and the constant current anemometer (CCA) have been traditionally used with hot wires for the study of turbulent flows. Several studies have been successfully conducted with them making important contributions in the understanding of turbulent flows. In recent years the patented ${ }^{1}$ constant voltage anemometer (CVA) is also emerging as a good alternative tool for applications in fluid dynamics. Several experimental studies have been conducted with the CVA in boundary layer transition in hypersonic flows ${ }^{2-4}$ at NASA Langley Research Center. A very good comparative study of the CTA and CVA was also carried out with experiments in a probe calibration wind tunnel. ${ }^{5}$ The frequency response of the CVA and CTA were also studied using laser radiative heating of the hot wire in an air jet. ${ }^{6}$ Analysis relating the sensitivity coefficients of the three anemometers (CTA, CCA, and CVA) and the time constants relationship of the CVA and CCA was recently presented by Comte-Bellot. ${ }^{7}$ In addition, the CVA was successfully used with hot films to detect the incidence of the transonic shock in flight on an F-15B aircraft. ${ }^{8} \mathrm{CVA}$ had also been used in under water application with hot films to detect transition and flow bifurcation regions on a hydrofoil. ${ }^{9}$ Some of the features noticed in these

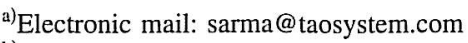

b)Electronic mail: gcb@selene.mecaflu.ec-lyon.fr

c)Electronic mail: faure@iris6.lemfi.u-psud.fr
}

studies are lower electromagnetic interference noise in CVA compared to the other anemometers, very small variation of the bandwidth from changes with the hot wire overheat and Reynolds number, and tolerance of larger cable lengths without affecting the bandwidth. One of the most important aspects of the CVA operation is the appropriate compensation for the thermal inertia of the hot wire (which manifests as a first-order time constant) to extend the bandwidth of the CVA. It is shown in the present article that with the CVA it is possible to set a fixed hardware compensation for the time constant (TC), measure the in situ time constant of the wire, and apply the necessary correction to the fixed compensation setting for the actual measured time constant (through software processing) to obtain accurate results. This approach is possible with the CVA because of its well-defined circuit behavior without any influence of the external hot wire cable capacitance. Productivity of the measurements is also enhanced with this method. In the following sections the application of this method for turbulence measurements in the boundary layer of a supersonic wind tunnel is presented.

The CVA circuit was analyzed by Sarma in terms of its transfer function in Ref. 10 where a method of measuring the in situ TC of the hot wire under constant voltage (CV) operation was also described. To demonstrate the versatility of the method, two fixed compensation settings ( 0.2 and 0.4 $\mathrm{ms}$ ) were used for taking data at the same point in addition to three overheat settings. The output voltage at each test point was then corrected through software in the postprocessing of 
the data with the in situ measured TC of the hot wire. In the experiments reported here TC was measured with quick manual adjustments on a digital scope and data was postprocessed to calculate the TC. This adjustment has since been fully automated in the CVA, thus eliminating all manual adjustments during the entire experimental process, a feature particularly useful for blow-down tunnels. It is also shown that this procedure assures a constant bandwidth for all the tests with a particular hardware compensation setting. The processed voltage perturbations from the CVA were converted to mass flux and temperature components using the sensitivity expressions derived by Comte-Bellot in Ref. 7.

\section{HARDWARE DESIGN}

\section{A. Hot wire time constant}

The dynamic behavior of the hot wire can be described through a differential equation:

$$
M_{\mathrm{CVA}} \frac{d r}{d t}+r=a i+b u,
$$

where $M_{\mathrm{CVA}}$ is the TC of the hot wire under CV operation, $r$ is the perturbation in the hot wire resistance, $i$ and $u$ are the perturbations in the hot wire current and flow velocity, respectively, and $a$ and $b$ are calibration constants. With $u$ $=0, M_{\mathrm{CVA}}$ can be determined from the first-order response due to a step change in the hot wire current. Typical averaged responses from the measurement of the TC in the supersonic tunnel for a 2.5 and $5 \mu \mathrm{m}$ hot wires are shown in Figs. 1(a) and 1(b), respectively. Numerical values of the $M_{\mathrm{CVA}}$ with various $V_{w}$ values obtained at $y / \delta=1.5$ at $M a_{\text {ext }}=2$ are shown in Fig. 2. Values of the time constants $M_{\mathrm{CCA}}$ under constant current $(\mathrm{CC})$ operation measured by Arzoumanian and Debieve ${ }^{11}\left(M a_{\text {ext }}=2.3\right)$ are also presented for quick comparison. It can be seen that while the hot wire TC increases with overheat under CC operation, it has quite the opposite effect under CV operation, just as predicted by Freymuth ${ }^{12}$ and in Ref. 7. The convergence of the TC values at higher overheats is apparent in Fig. 2. Also in Fig. 2, experimental data from the TC measurements in a low-speed tunnel on a $5 \mu \mathrm{m}$ hot wire with variations in hot wire overheat and its Reynolds number are shown. Figure 2 shows that the TC under the CV operation also converges to a constant value with higher Reynolds $(\mathrm{Re})$ numbers of the wire. Such a convergence to a constant value with the Reynolds number has been shown to be theoretically possible in an earlier study. ${ }^{13}$ The convergence of the TC to a constant value with higher Reynolds numbers and also with overheats (unlike CCA) of the wire will offer an immediate advantage of the CVA. There may be certain engineering applications in fluid mechanics involving measurements only above certain hot wire Reynolds numbers and overheat values, where it will thus be possible to use only a fixed compensation for the TC with a judicious $a$ priori choice of the value. Thus, adjustments for each test point are eliminated for that application. The reduction of the hot wire TC under CV operation relative to the CC operation makes the CVA operate with a smaller ac gain from the compensation circuit than the CCA for the same bandwidth.

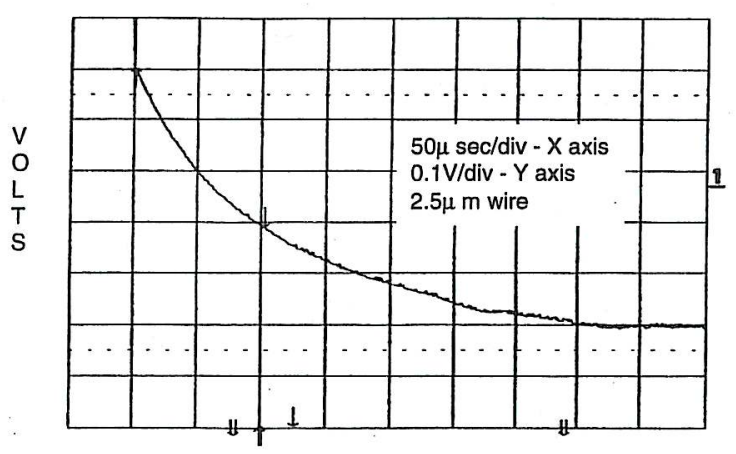

(a)

Time
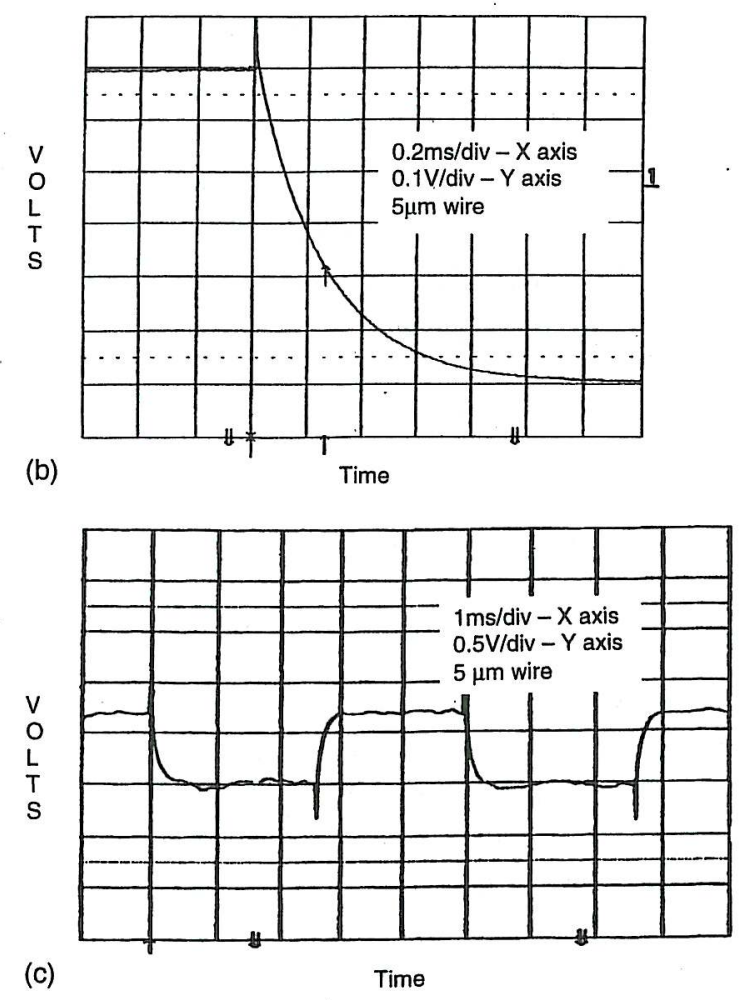

FIG. 1. (a) Averaged step response of a $2.5 \mu \mathrm{m}$ hot wire with overheat $a_{w}=0.5$ at $y / \delta=1.5$ at $M a_{\text {ext }}=2.0$. (b) Averaged step response of a $5 \mu \mathrm{m}$ hot wire with overheat $a_{w}=0.6$ at $y / \delta=1.5$ at $M a_{\mathrm{ext}}=2.0$. (c) Typical output from TC measurement unit of the CVA with a $5 \mu \mathrm{m}$ wire with an overheat $a_{w}=0.5$ at $y / \delta=1.5$ at $M a_{\mathrm{ext}}=2.0$.

\section{B. Compensated CVA}

A compensated CVA circuit is shown in Fig. 3. The transfer function of the circuit as derived by Sarma in Ref. 10 is repeated below:

$$
\begin{aligned}
& \frac{e_{\mathrm{CVA}}}{u}(s) \\
& =\frac{\left(R_{2} / R_{w}\right) I_{w}}{\frac{s^{2}}{\omega_{n}^{2}}+\frac{\left(1 / T_{c}+R_{w} / R_{D} 2 \pi f_{t}\right)}{\omega_{n}^{2}} s+1} \frac{\left(1+T_{c} s\right)}{\left(1+M_{\mathrm{CVA}} s\right)} b^{\prime},
\end{aligned}
$$

where $e_{\mathrm{CVA}}$ and $u$ are the perturbations in CVA output and input velocity, respectively. $R_{2}=R_{a}+R_{b}$, and $R_{D}$ are components shown in Fig. 3 and $R_{w}$ is the hot wire resistance. $I_{w}$ is the mean hot wire current at the operating point that sets 


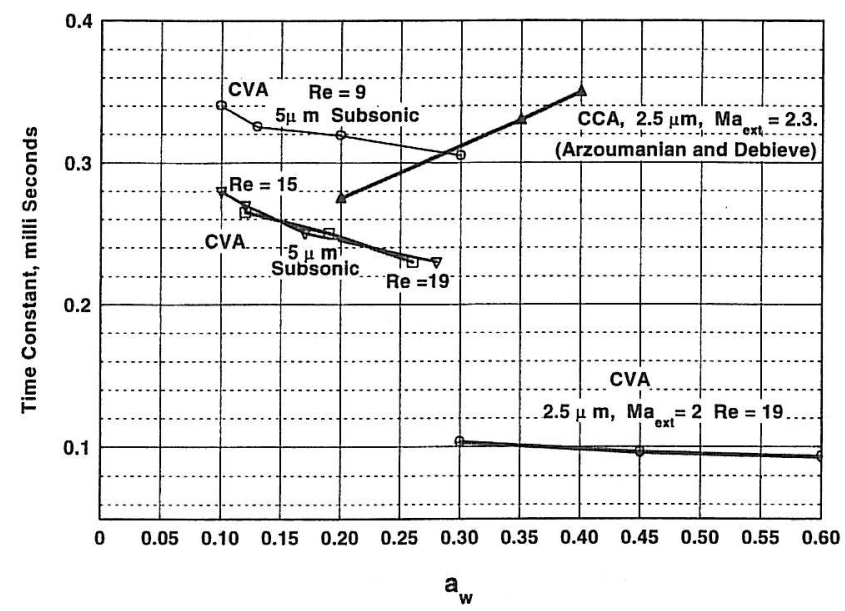

FIG. 2. Measured time constants of the hot wires at different overheat values $\left(a_{w}\right)$.

the overheat of the hot wire and $s$ is the Laplace operator. $f_{t}$ is the gain bandwidth product of the operational amplifier used in the CVA and $b^{\prime}$ is the calibration constant of the CVA and the hot wire. The other constant related to the circuit is $T_{c}$, the hardware time constant compensation setting, given by

$$
T_{c}=\frac{C R_{a} R_{b}}{R_{2}} .
$$

$\omega_{n}$ and $\zeta$ are defined in the next section.

The prototype CVA used in the present experiment is equipped with a variable compensation setting in 72 overlapping ranges covering a TC range of $0.1-0.6 \mathrm{~ms}$, and one way to conduct the test is to measure in situ $M_{\mathrm{CVA}}$ and select the matching $T_{c}$ in the CVA, thus canceling the pole with the zero in Eq. (2). However, the procedure for such a cancellation involves taking the hot wire $M_{\mathrm{CVA}}$ data, calculating the $M_{\mathrm{CVA}}$ at that point from the averaged data samples and manually setting the matching $T_{c}$ in the CVA. This process will impede the productivity of the test. With the complete postprocessing approach described in the next section, high productivity particularly attractive for blow-down tunnels can be assured with the CVA.

A possible test sequence with the CVA for higher productivity testing is formulated with the following steps:

(1) Position the hot wire in the desired location in the wind tunnel.

(2) Turn on the flow.

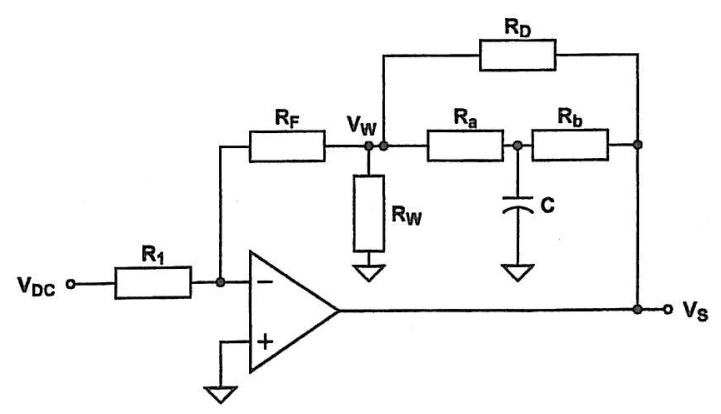

FIG. 3. Compensated CVA circuit.

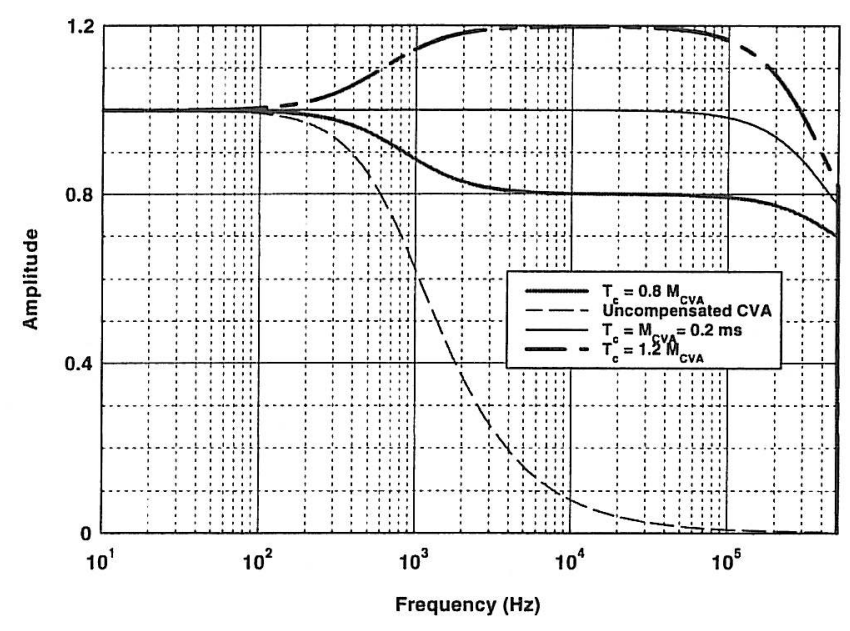

FIG. 4. Response curves due to the mismatch of the time constants.

(3) Adjust the desired overheat (remote adjustment and monitoring of the overheat is possible with the CVA).

(4) With the hot wire in the TC measurement mode of the CVA, obtain the $M_{\mathrm{CVA}}$ data for about $3 \mathrm{~s}$.

(5) Switch the hot wire to the turbulence measurement mode in the CVA (with a fixed compensation) from the TC measurement mode and take data for the desired duration.

\section{POSTPROCESSING}

\section{A. Time constant calculation}

The data obtained under step (4) in the previous section will be a series of first-order response pulses similar to those shown in Fig. 1(c). The average of such a series of pulses are shown in Figs. 1(a) and 1(b) for a 2.5 and $5 \mu \mathrm{m}$ hot wires, respectively. The $M_{\mathrm{CVA}}$ of the hot wire is calculated as the time it takes the amplitude to reach $63 \%$ of the final value from the initial value. Measured values of the time constants $\left(M_{\mathrm{CVA}}\right)$ in the present experiment are shown in Fig. 2.

\section{B. Software correction and constant bandwidth operation}

The data from CVA could be collected by using a fixed $T_{c}$ setting, while also collecting the $M_{\mathrm{CVA}}$ data at each test point without actually matching them at the time of the test. Figure 4 shows typical outputs that would arise due to mismatch of the $T_{c}$ and $M_{\mathrm{CVA}}$. In postprocessing, $M_{\mathrm{CVA}}$ is computed from the acquired TC measurement data and correction can be applied to the set $T_{c}$. The CVA output voltage is modified by the following equation:

$$
e_{c}=e_{\mathrm{CVA}}\left[\frac{1+M_{\mathrm{CVA}} s}{1+T_{c} s}\right],
$$

where, $e_{\mathrm{CVA}}$ is the acquired CVA output and $e_{c}$ is the corrected output of the CVA. It is seen that Eq. (4) cancels the fixed compensation setting $T_{c}$ used in the CVA and applies the correct compensation value from the measured $M_{\mathrm{CVA}}$, thereby canceling the denominator term with the numerator term in Eq. (2). The bracketed expression in Eq. (4) is implemented with software and applied to the sampled analog 
voltages from the CVA output. The CVA without any hardware compensation (full software compensation) is not a good approach since the signal levels from such a system would be low, even though its bandwidth could be several times larger than a hardware compensated CVA. The limiting case for the bracketed expression in Eq. (4) is $\left(M_{\mathrm{CVA}} / T_{c}\right)$, and is independent of frequency.

When the pole due to the hot wire time constant is canceled by the zero due to the compensating circuit ( $M_{\mathrm{CVA}}$ $=T_{c}$ ) the transfer function in Eq. (2) shows that the CVA will now be a simple second-order system as given below:

$$
\frac{v_{s}}{u}(s)=\frac{\left(R_{2} / R_{w}\right) I_{w}}{s^{2} / \omega_{n}^{2}+\left[\left(1 / T_{c}+R_{w} / R_{D} 2 \pi f_{t}\right) / \omega_{n}^{2}\right] s+1} b^{\prime} .
$$

In Ref. 10 , it was shown that the natural frequency $\omega_{n}$ and the damping ratio $\zeta$ of the above transfer function are given by

$$
\omega_{n}=\sqrt{\frac{R_{w}}{R_{2}} \frac{2 \pi f_{t}}{T_{c}}\left(1+\frac{R_{2}}{R_{D}}\right)}
$$

and

$$
\zeta=\frac{1}{2} \frac{\left(\frac{1}{T_{c}}+2 \pi f_{t} \frac{R_{W}}{R_{D}}\right)}{\sqrt{\frac{R_{w}}{R_{2}} \frac{2 \pi f_{t}}{T_{c}}\left(1+\frac{R_{2}}{R_{D}}\right)}},
$$

where $R_{w}, R_{2}$ are as shown in Fig. 3. The bandwidth (BW) of such a system can be estimated from the following equation:

$$
\mathrm{BW}=\omega_{n} \sqrt{\left(1-2 \zeta^{2}\right)+\sqrt{4 \zeta^{4}-4 \zeta^{2}+2}} .
$$

In the above equations [Eqs. (6), (7) and (8)], for a given design, the only variables that could influence the BW are $R_{w}$ and $T_{c}$ and the $B W$ is independent of the cable capacitance. Even so, with the software compensation approach, $T_{c}$ is also held constant, leaving only one variable $R_{w}$ that may have any effect on the $\mathrm{BW}$ at all. Variable $R_{w}$ means variable overheat. In Ref. 5 it was experimentally established that in the CVA, the BW does not change with overheat, which was theoretically substantiated in Ref. 10. As shown in Ref. 10, an increase in $R_{w}$ increases both $\omega_{n}$ and $\zeta$ to make Eq. (8) fairly constant. A lower $T_{c}$ would, of course, give a larger BW. In the present experiments, two compensation settings at 0.2 and $0.4 \mathrm{~ms}$ were used, giving 230 and $120 \mathrm{kHz}$ bandwidths, respectively. It may appear attractive to select a lower compensation setting for $T_{c}$ to increase the bandwidth, but unless it is warranted by the test requirements such a selection of a larger bandwidth may also increase the system noise. The biggest advantage with this method is, therefore, that the bandwidth remains constant for the test with higher productivity.

\section{WIND TUNNEL TESTING AND DATA PROCESSING}

Turbulence measurements were made in the Mach 2 blow-down tunnel of the CEAT in Poitiers (France). The conditions at the upstream of the sonic throat are typically
$285 \mathrm{~K}$ and $9.5 \times 10^{4} \mathrm{~Pa}$. The turbulent boundary layer on the flat floor of the $150 \mathrm{~mm} \times 150 \mathrm{~mm}$ test section was used for the test. At the downstream location of the measurements the boundary layer thickness $\delta$ was $15.7 \mathrm{~mm}$, and the mean and turbulence characteristics have been fully documented by Dury. ${ }^{14}$ In the present study, most of the turbulence measurements were made at a wall distance $y$ corresponding to $y / \delta$ $=0.64$. A few additional measurements were also taken at $y / \delta=0.32$. A $0.5 \mathrm{~mm}$ length, $2.5 \mu \mathrm{m}$ diam tungsten hot wire with a cold resistance $R_{a} \simeq 6 \Omega$ was used. The cable resistance was $\simeq 0.9 \Omega$ and this value was taken into account to obtain $R c_{a}$ (cold resistance of the wire placed in the flow), $R_{w}$ (heated wire resistance), and the associated overheat ratio $a_{w}=\left(R_{w}-R c_{a}\right) / R c_{a}$. Three overheat ratios, i.e., $0.30,0.45$, and 0.62 were generally used. All the turbulence signals were recorded with a sampling rate of $10^{6} \mathrm{samples} / \mathrm{s}$ for about $0.25 \mathrm{~s}$ using a 16 bit analog-to-digital converter and stored in binary format in a workstation. They were later converted to ASCII format for analysis. Data were processed in terms of 4096 points and 29 such samples were averaged for the final results. The $M_{\mathrm{CVA}}$ values were measured in situ outside the boundary layer at $y / \delta=1.5$ where the free stream turbulence was about $1 \%$. The time constant values remained fairly constant throughout the external part of the boundary layer because the wire $\operatorname{Re}$ remained fairly large for $y / \delta$ $=0.32$ to 1.5 with the wire $\operatorname{Re}$ in the range $13.5-19$, respectively. The measured $M_{\mathrm{CVA}}$ values in this tunnel at three overheats $\left(\bar{a}_{w}\right)$ have been already shown in Fig. 2 . They range from $0.103 \mathrm{~ms}$ at an overheat of $0.30-0.096 \mathrm{~ms}$ at an overheat of 0.45 and $0.093 \mathrm{~ms}$ at an overheat of 0.62 . Spurious low-frequency unsteady flow was inherent in the supersonic flow in the wind tunnel generated by a downstream ejector powered by a 110-160 bar air supply. These lowfrequency signals were filtered out by using a high-pass (hp) filter with a $3 \mathrm{kHz}$ cutoff. This hp filtering was also implemented by Dury ${ }^{14}$ who has extensively studied the tunnel performance and found that the filtering did not affect the turbulence levels. As a check, the integral length scales $L$ were calculated from the spectra and are found to be within the correct range $L / \delta \simeq 0.2-0.3$, agreeing with previous data, e.g., Smits and Dussauge. ${ }^{15}$ Unless otherwise identified, the data collected with $T_{c}=0.2 \mathrm{~ms}$ for which that prototype CVA had a $230 \mathrm{kHz}$ bandwidth, were filtered with a lowpass (lp) filter with a cutoff at $120 \mathrm{kHz}$, making it equal to the bandwidth obtained with the $T_{c}=0.4 \mathrm{~ms}$ setting of the CVA. (The CVA has since been improved to have over 300 $\mathrm{kHz}$ at $T_{c}=0.2 \mathrm{~ms}$.) This low-pass filtering also brought the CVA bandwidth closer to the CTA bandwidth used for comparison at the point at $y / \delta=0.32$.

\section{RESULTS}

\section{A. Effect of a small change in $M_{\text {cva }}$ at a compensation setting $T_{c}$}

With the CVA compensation TC set at $T_{c}=0.2 \mathrm{~ms}$, the power spectral density (psd) plots were calculated for $M_{\mathrm{CVA}}=0.095,0.100$, and $0.105 \mathrm{~ms}$, a $5 \%$ change on either side of a nominal value $0.100 \mathrm{~ms}$ at an overheat of 0.62 . The $5 \%$ change in $M_{\mathrm{CVA}}$ is larger than the $\pm 2 \%$ estimated maxi- 


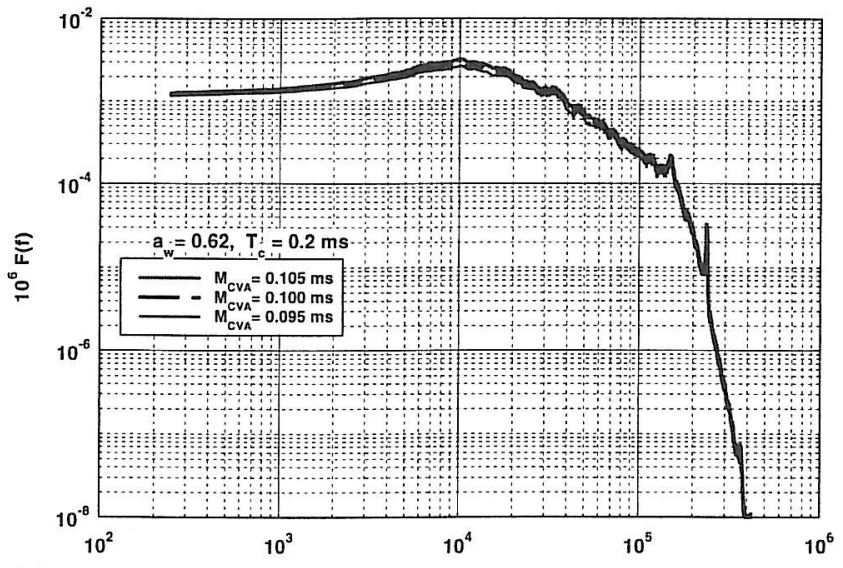

(a)

Frequency, $f(\mathrm{~Hz})$

$\ln f / 2.3$

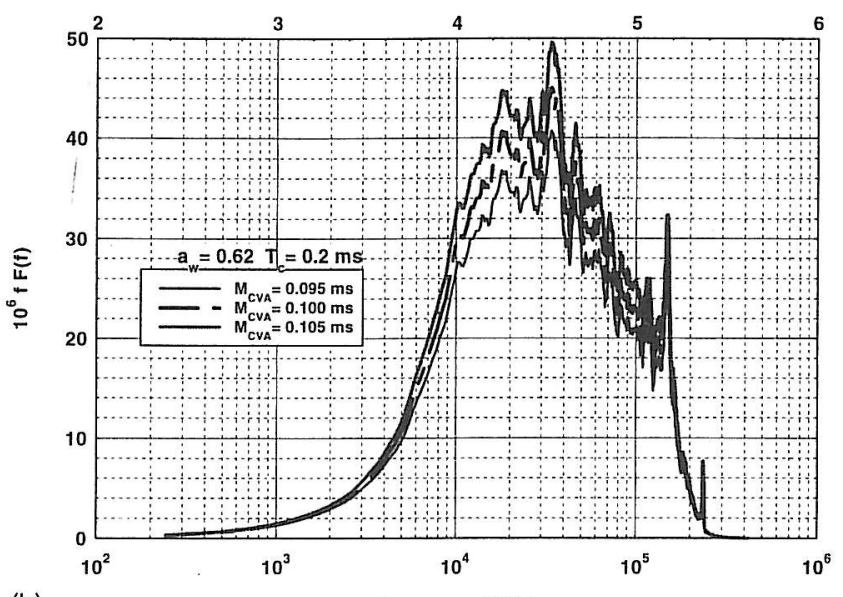

(b)

Frequency, $f(\mathrm{~Hz})$

FIG. 5. (a) Response changes in $F(f)$ due to variations in $M_{\mathrm{CVA}}$. (b) Response changes in $f F(f)$ due to variations in $M_{\mathrm{CVA}}$.

mum error in the TC measurement process. The $\left(e^{\prime} / E\right)$ psd curves so obtained are plotted as $F(f)$ in Fig. 5(a). It can be seen that all three curves are close to each other with an error of $\pm 4 \%$ in the rms values of the fluctuating voltage. A more physically meaningful and more stringent comparison would be to invoke the definition:

$$
\frac{e^{\prime 2}}{E^{2}}=\int_{0}^{\infty} F(f) d f
$$

from which we can obtain:

$$
\frac{e^{\prime 2}}{E^{2}}=\int_{0}^{\infty} f F(f) \frac{d f}{f}=\int_{0}^{\infty} f F(f) d(\ln f) .
$$

Hence, if we plot $f F(f)$ on the $Y$ axis and $\ln f$ on the $X$ axis, we can have linear scales for both. This plot is shown in Fig. 5(b). By simple visual inspection alone, significant features of the signals will be apparent, like the most energetic frequency range of the turbulent signal and the absolute differences in different spectra.

\section{B. Comparison of two compensation settings}

One important consideration for the software correction should be its adaptability to apply to any reasonable compen-
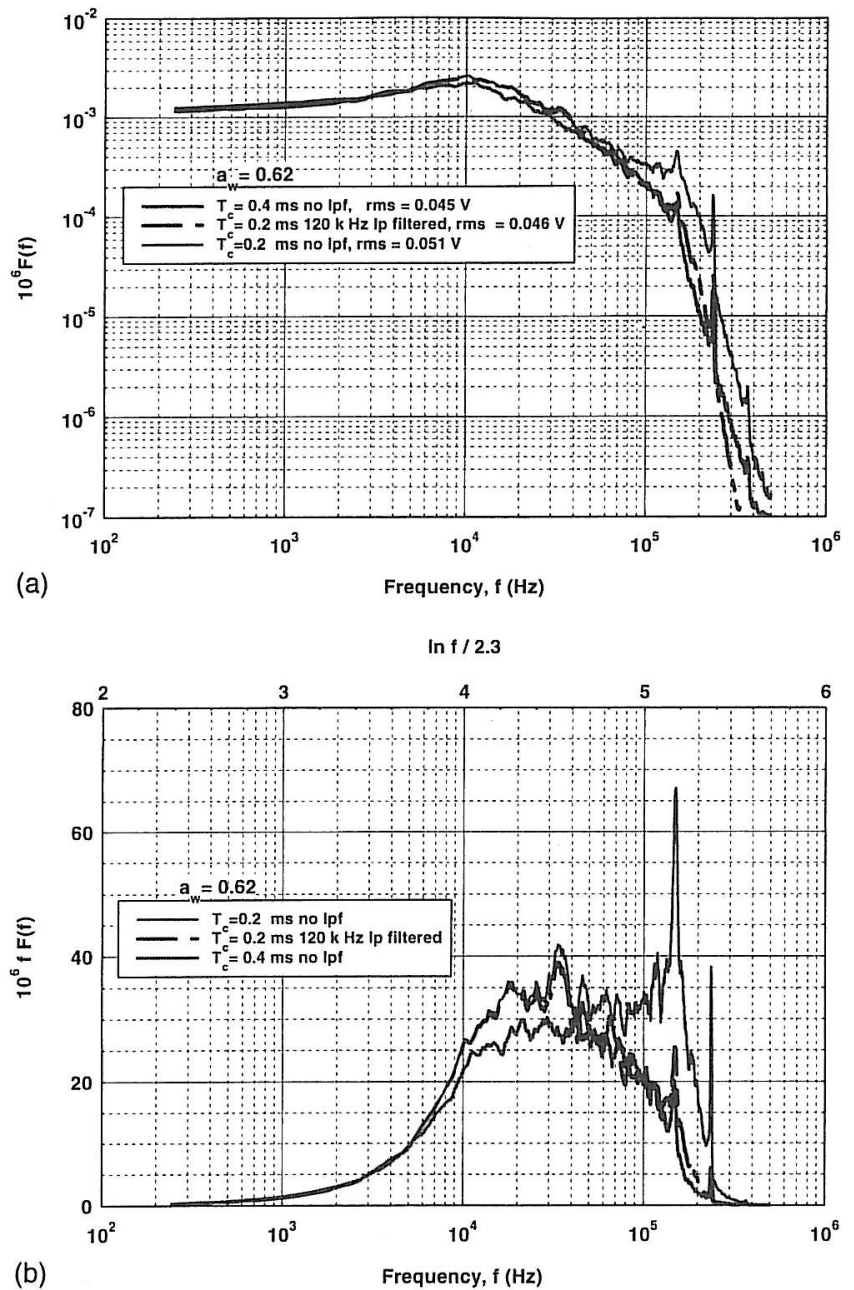

FIG. 6. (a) Response from two compensation settings in $F(f)$ at $a_{w}$ $=0.62$. (b) Response from two compensation settings in $f F(f)$ at $a_{w}$ $=0.62$.

sation setting. That is, if a CVA with only one fixed compensation setting $\left(T_{c}\right)$ can be used for a wide range of measurements, then the hardware design becomes simple and a specified bandwidth can be assured. To study such a case, data from two $T_{c}$ settings $(0.2$ and $0.4 \mathrm{~ms})$ at an overheat of 0.62 was processed for psd with the software correction of the actual measured $M_{\mathrm{CVA}}=0.093 \mathrm{~ms}$. For the $0.2 \mathrm{~ms}$ setting, a $120 \mathrm{kHz}$ lp filter (lpf) was again used. The corresponding plots are shown in Figs. 6(a) and 6(b). From the legend on the graph and the plots, it can be seen that there is good agreement between the two data sets $\left(T_{c}\right)$, indicating that indeed the software correction is accurate. The peaks around 150 and $250 \mathrm{kHz}$ are due to strain gauging of the hot wire similar to that explained by Smits and Dussauge. ${ }^{15}$ Due to the bandwidth limit of the $0.4 \mathrm{~ms}$ setting, the strain gauge peaks are less visible for that case. The $120 \mathrm{kHz}$ lp filter used with the $0.2 \mathrm{~ms}$ setting to take its bandwidth closer to the 0.4 ms setting, also leads to a reduction in the strain gauging peaks in the former setting. This filtering does not affect the kinetic-energy content of the turbulence. Kistler ${ }^{16}$ has shown that supersonic turbulence spectra encompass energetic eddy sizes down to one-fourth of the boundary layer thickness, $\left(f_{\max } \delta / 4\right) / U \simeq 1$. In the present case, the above condition 


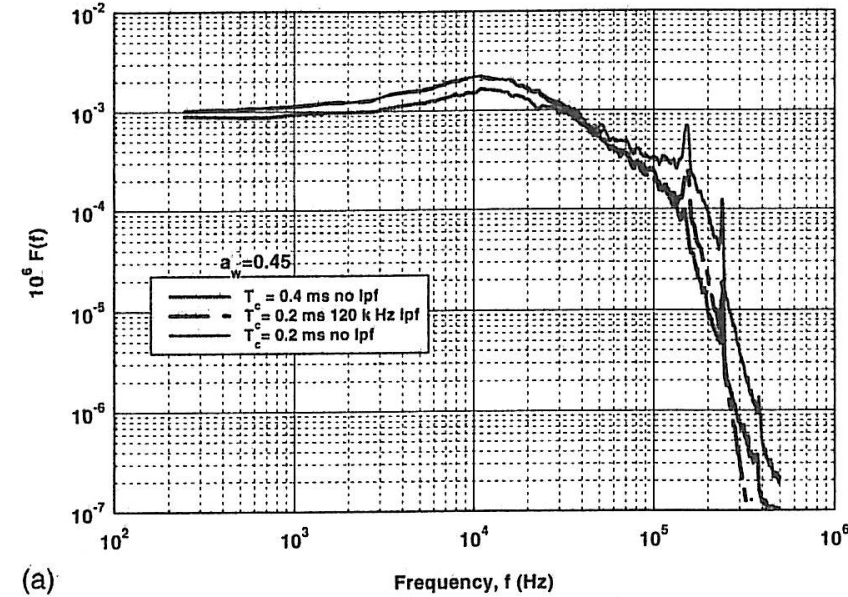

(a)

Frequency, $f(\mathrm{~Hz})$

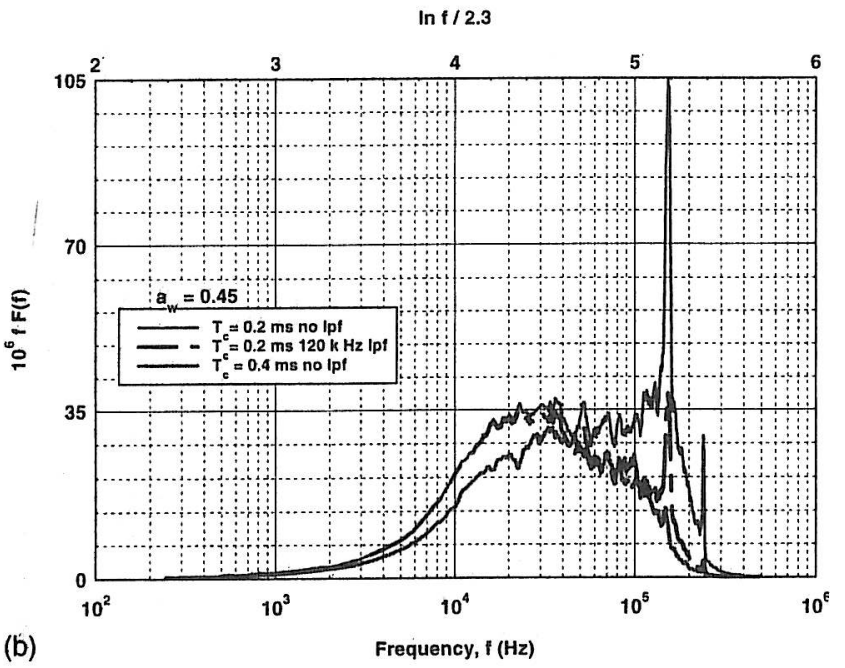

FIG. 7. (a) Response changes in $F(f)$ due to $T_{c}$ changes at $a_{w}=0.45$. (b) Response changes in $f F(f)$ due to $T_{c}$ changes at $a_{w}=0.45$.

gives a $f_{\max }=100 \mathrm{kHz}$, so that the $120 \mathrm{kHz}$ lp filter of the data should be acceptable. Again, the agreement between the two compensation settings shown in Figs. 6(a) and 6(b) is an indication of the versatility of the CVA setup and software compensation method. Figures $7(\mathrm{a})$ and $7(\mathrm{~b})$ show results similar to Fig. 6, except now it is with an overheat of 0.45 .

\section{Comparison of CVA and CTA spectra}

A Dantec CTA (M5012) was used to collect the turbulence data with the frequency response adjusted with a gain setting of 4 , high-pass filter 2 . The maximum bandwidth with such a setting was specified as $100 \mathrm{kHz}$. The data were collected with the wire located at $y / \delta=0.32$ with an overheat of

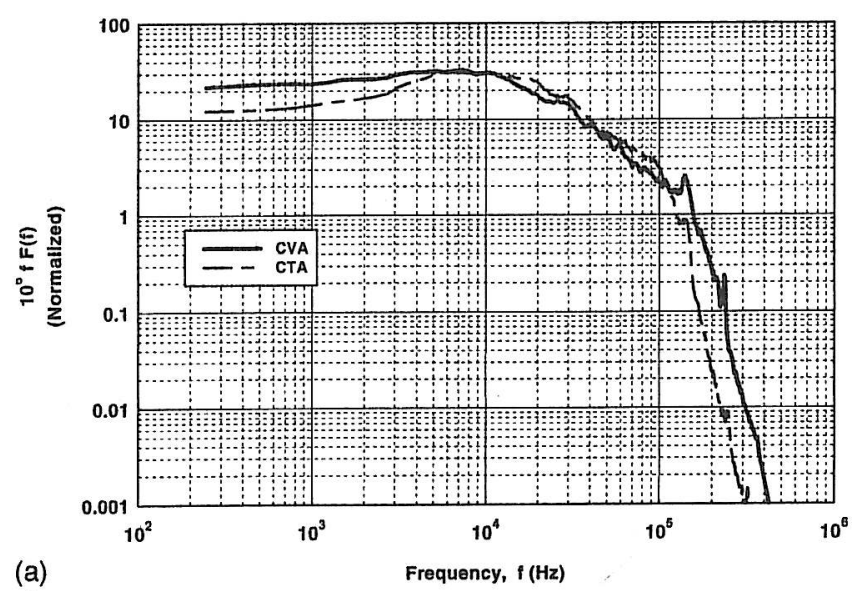

$\ln f / 2.3$

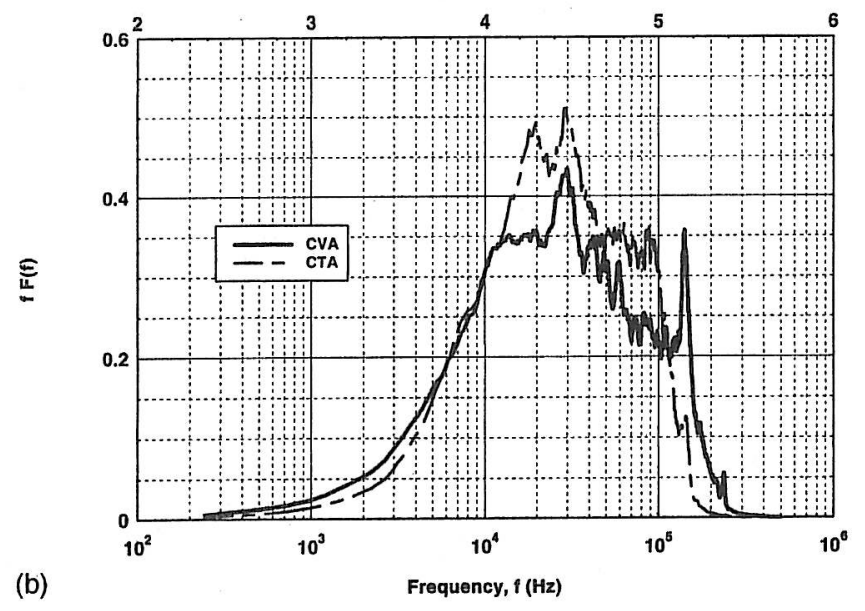

FIG. 8. (a) $F(f)$ Spectra of CVA and CTA normalized with their meansquare output voltages. (b) $f F(f)$ responses in CVA and CTA normalized with their mean-square output voltages.

0.60. The mean and rms voltages of the CTA are 2.21 and $0.065 \mathrm{~V}$, respectively. With the same overheat setting, data were also collected at the same point with the CVA with $T_{c}=0.2 \mathrm{~ms}$, cf. Table I. Psd plots from both are shown in Figs. 8(a) and 8(b). The anemometer psds were normalized with their respective mean-square outputs. Very distinct peaks at 150 and $250 \mathrm{kHz}$ due to the aforementioned strain gauging effect are observed in the CVA but not as much in the CTA. Figure 8 (b) shows broader energy levels in the CVA than the CTA because of the larger bandwidth in the CVA even with the lp filter. From Fig. 8(b) it can be seen that energy contributions below $5 \mathrm{kHz}$ are insignificant. In the most energetic part of the spectrum $(5-100 \mathrm{kHz})$ the

TABLE I. Summary of CVA test conditions and results.

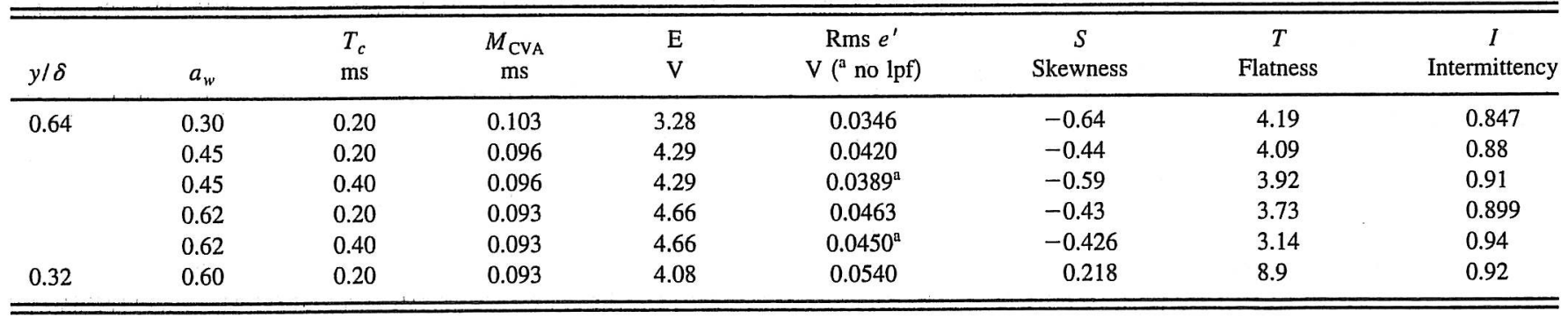


response from both the anemometers is very similar. The differences below $5 \mathrm{kHz}$ are attributed to remnants of wind tunnel unsteadiness.

\section{Skewness, flatness, and intermittency factors from CVA}

Table I summarizes the various test points using the CVA in the present experiments in the supersonic tunnel. It also presents the rms output voltage, the skewness, the flatness factors, and the intermittency factors at each test run. Results show that the majority of cases have a negative skewness factor around -0.4 , which agrees with the results obtained by Dury ${ }^{14}$ in the same tunnel. The negative skewness suggests negative spikes in the turbulent signal. The intermittency factor with a $10 \mathrm{mV}$ threshold is between 0.84 and 0.94 .

\section{E. Mass flux and temperature characteristics of the turbulence}

A hot wire responds to both mass flux and total temperature fluctuations in the turbulence with relative sensitivity depending upon the overheat of the wire, cf. Kovasznay. ${ }^{17}$ For small perturbations in mass flux $\rho u$ and temperature $\theta_{t}$ we can write the wire response using the wire sensitivity coefficients $S_{\rho u}^{\mathrm{CVA}}$ and $S_{\theta_{t}}^{\mathrm{CVA}}$ as

$$
\frac{e^{\prime}}{E}=S_{\rho u}^{\mathrm{CVA}} \frac{(\rho u)^{\prime}}{\bar{\rho} U}+S_{\theta_{t}}^{\mathrm{CVA}} \frac{\theta_{t}^{\prime}}{\Theta_{t}} .
$$

In principle, values of the sensitivity coefficients could be evaluated through the calibration of the hot wire. Fortunately, however, because of knowledge of the CCA and CTA systems, the sensitivity coefficients of the CVA can be evaluated knowing the CVA setup fairly well, cf. Comte-Bellot. ${ }^{7}$ These can be expressed as (from Ref. 7):

$$
S_{\rho u}^{\mathrm{CVA}}=S_{\rho u}^{\mathrm{CTA}}\left[\frac{2 a_{w}}{\left(1+2 a_{w}\right)}\right] \text { with } S_{\rho u}^{\mathrm{CTA}} \simeq 0.25
$$

and

$$
S_{\theta_{t}}^{\mathrm{CVA}}=-S_{\theta_{t}}^{\mathrm{CCA}}\left[\frac{1}{\left(1+2 a_{w}\right)}\right] \text { with } S_{\theta_{t}}^{\mathrm{CCA}} \simeq 1 .
$$

In the above expressions for the CVA, the advantage that $S_{\rho u}^{\mathrm{CTA}}$ and $S_{\theta_{t}}^{\mathrm{CCA}}$ are nearly constant with $a_{w}$ is utilized. Squaring and averaging Eq. (11) gives

$$
\begin{aligned}
\overline{\frac{e^{\prime 2}}{E^{2}}=} & {\left[S_{\rho u}^{\mathrm{CVA}}\right]^{2} \frac{\overline{(\rho u)^{\prime 2}}}{\bar{\rho}^{2} U^{2}}+2\left[S_{\rho u}^{\mathrm{CVA}} S_{\theta_{t}}^{\mathrm{CVA}}\right] \overline{\frac{(\rho u)^{\prime} \theta_{t}^{\prime}}{\bar{\rho} U \Theta_{t}}} } \\
& +\left[S_{\theta_{t}}^{\mathrm{CVA}}\right]^{2} \frac{\overline{\theta_{t}^{2}}}{\Theta_{t}^{2}} .
\end{aligned}
$$

Equation (14) is valid for a fixed overheat ratio and has to be solved for the three unknowns $\overline{(\rho u)^{\prime 2}} / \bar{\rho}^{2} U^{2}, \overline{(\rho u)^{\prime} \theta_{t}^{\prime}} /$ $\bar{\rho} U \Theta_{t}$, and $\overline{\theta^{\prime 2}} / \Theta_{t}^{2}$. To solve for the three unknowns, we need to obtain data at three overheat values at the test point. To accomplish this, the present experiment was conducted with the three overheat values of $0.30,0.45$, and 0.62 with $T_{c}=0.2 \mathrm{~ms}$ and $120 \mathrm{kHz} \mathrm{lpf}$, at $y / \delta=0.64$. The $S_{\rho u}^{\mathrm{CTA}}$ value

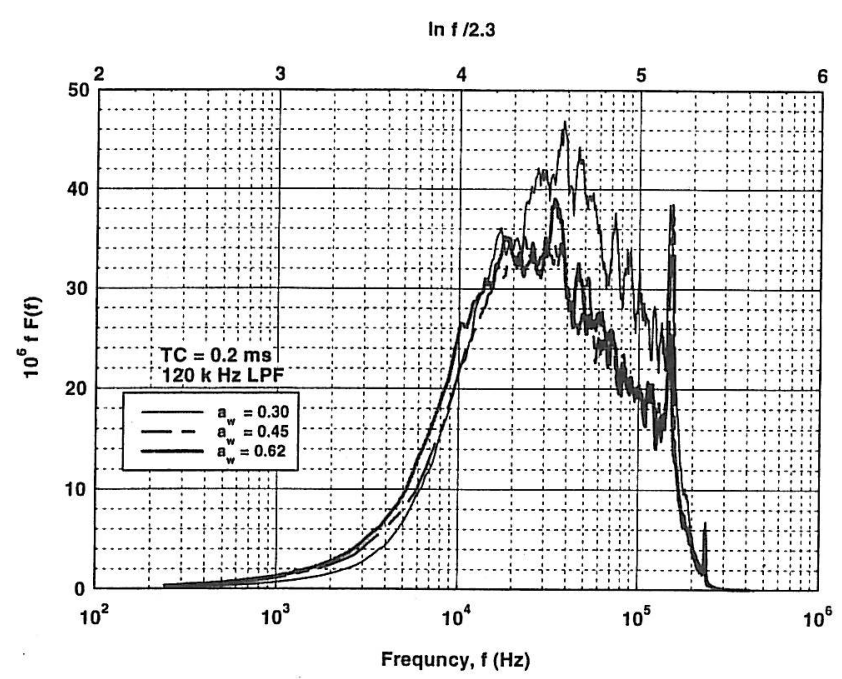

FIG. 9. CVA response of $f F(f)$ at three overheats.

was more like 0.22 instead of 0.25 according to Dury's work. ${ }^{14}$ Using the rms values listed in Table I, the components in the turbulence are calculated to be

$$
\frac{\sqrt{\overline{(\rho u)^{\prime 2}}}}{\bar{\rho} U}=8.87 \%, \quad \frac{\sqrt{\overline{\theta_{t}^{\prime 2}}}}{\Theta_{t}}=1.93 \%,
$$

and

$$
R=\frac{\overline{(\rho u)^{\prime} \theta_{t}^{\prime}}}{\sqrt{\overline{(\rho u)^{\prime 2}}} \sqrt{\overline{\theta_{t}^{\prime 2}}}}=0.49
$$

The same technique could be applied to the power spectral density calculations as well. Figure 9 shows the plots of the $e^{\prime} / E$ spectra at $T_{c}=0.2 \mathrm{~ms}$ with $120 \mathrm{kHz}$ lpf solved from the three overheats $0.30,0.45$, and 0.62 . The $f F(f)$ versus $\ln (f)$ shown in Fig. 9 gives a clear glimpse of the most energetic part of the turbulent signals. The three responses are different, as they should be at different overheats. Using the three data arrays for the three overheats, the matrix solution of the spectra is obtained for $G_{\rho u}(f)=\operatorname{psd}(\rho u)^{\prime}$, defined by $\overline{(\rho u)^{\prime 2}} / \bar{\rho}^{2} U^{2}=\int_{0}^{\infty} G_{\rho u}(f) d f$ and $G_{\theta_{t}}(f)=\operatorname{psd}\left(\theta_{t}^{\prime}\right)$ defined by $\overline{\theta_{t}^{\prime 2}} / \bar{\Theta}_{t}^{2}=\int_{0}^{\infty} G_{\theta_{t}}(f) d f$, and the cross-correlation spectra is denoted by $G_{\rho u \theta}$. These spectra are shown in Fig. 10(a). As can be expected ${ }^{15}$ the total temperature fluctuation component is small. The psd of the correlation coefficient $R(f)$ is shown in Fig. 10(b). The numerical value of the $R(f)$ computed with this method is smaller than 1 , except in the vicinity of the strain gauging peaks, which are difficult to analyze anyway. The above results demonstrate that it is possible to use the CVA also with different overheats and resolve the turbulence components.

\section{F. Comparison with earlier research}

An exhaustive chart of previously measured turbulence intensities by different researchers was presented by Smits, Hayakawa, and Muck. ${ }^{18}$ In order to put the present CVA data into that chart, we need to transform the $\overline{(\rho u)^{\prime 2}} / \bar{\rho}^{2} U^{2}$ results into $\bar{\rho} \overline{u^{\prime 2}} / \tau_{*}$, where $u^{\prime}$ is the velocity fluctuation, $\bar{\rho}$ is the 


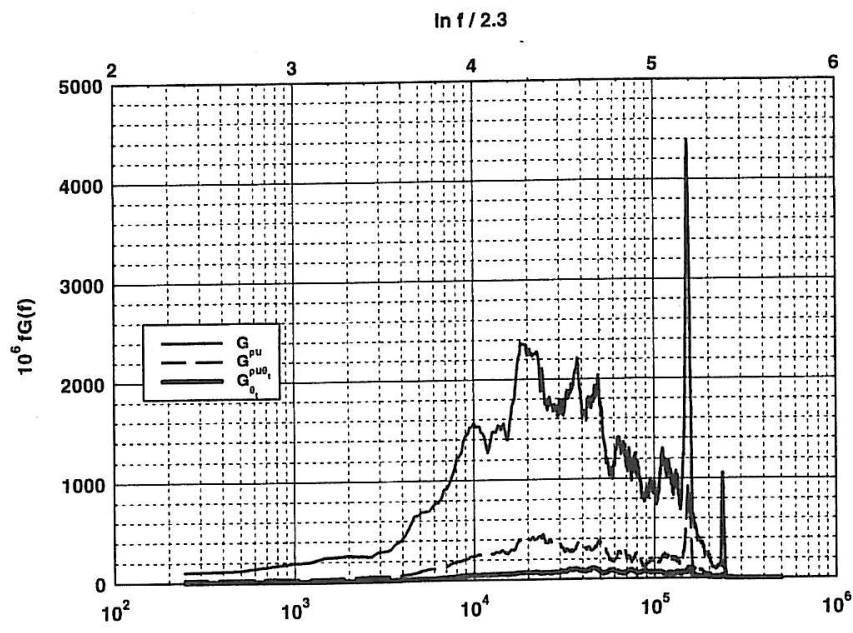

(a)

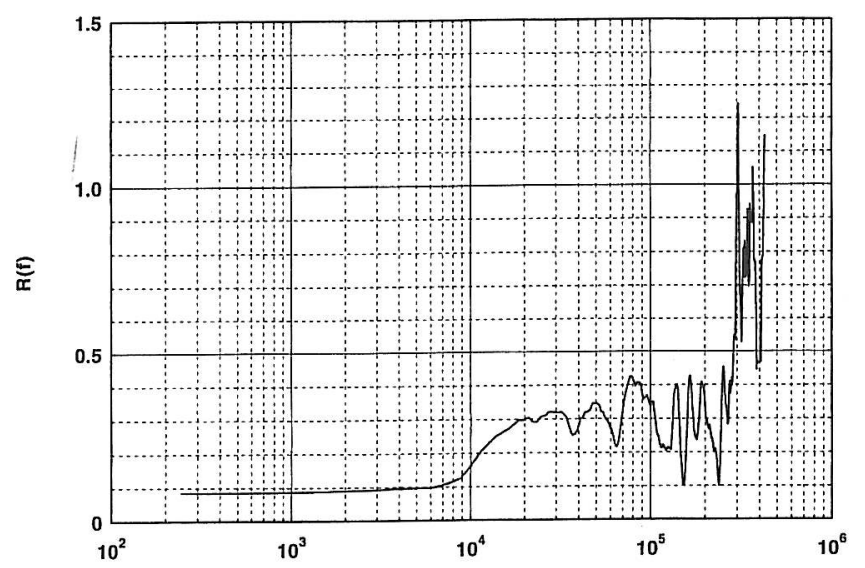

(b)

Frequency, $f(\mathrm{~Hz})$

FIG. 10. (a) Resolved components of turbulence obtained by solving three simultaneous equations with three overheats. (b) Spectra of the correlation coefficient $R(f)$ for CVA.

mean local density, and $\tau_{*}$ is the wall stress such that $\tau_{*}$ $=\rho_{*} u_{*}^{2}$ where $\rho_{*}$ is the density at the wall and $u_{*}$ is the friction velocity. Several of the characteristics of the supersonic tunnel needed to convert the CVA output into $\bar{\rho} \overline{u^{\prime 2}} / \tau_{*}$ for comparison with earlier research are available from Dury's work $^{14}$ in the same tunnel: $u_{*}=20 \mathrm{~m} / \mathrm{s}, \rho_{*}$ $=0.1455 \mathrm{~kg} / \mathrm{m}^{3}, \quad M a_{\text {ext }}=2, \quad U_{\text {ext }}=505 \mathrm{~m} / \mathrm{s}$, $\rho_{\text {ext }}=0.2682 \mathrm{~kg} / \mathrm{m}^{3}$, and $\operatorname{Re}_{\text {ext }}=19$. Profiles of the local wire Reynolds number Re and the flow Mach number $M a$ across the boundary layer were also available, from which, at $y / \delta$ $=0.64, \mathrm{Re}=17$ and $M a=1.8$.

The first step in the computations is to transform the relative change $(\rho u)^{\prime} / \bar{\rho} U$ into

$$
\frac{(\rho u)^{\prime}}{\bar{\rho} U}=\frac{\rho^{\prime}}{\bar{\rho}}+\frac{u^{\prime}}{U} .
$$

It is assumed that the static pressure across the boundary layer and stagnation temperature are constants. It was also assumed that the strong Reynolds analogy (SRA) is valid for the test, cf. Gaviglio ${ }^{19}$ and Barre, Dupont, and Dussauge ${ }^{20}$ so that:

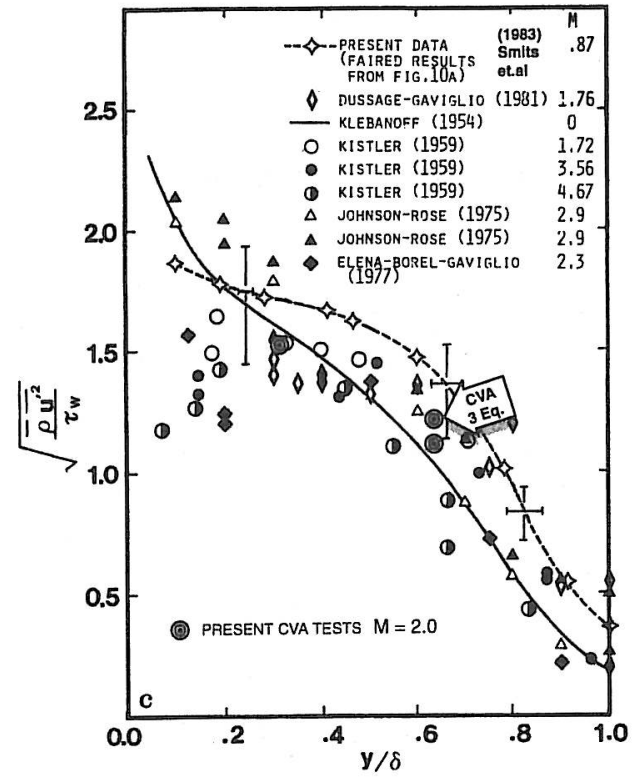

FIG. 11. CVA test results superimposed with earlier research results.

$$
(\gamma-1) M a^{2} \frac{\left(\overline{u^{\prime 2}}\right)^{1 / 2}}{U} \simeq \frac{\left(\overline{\rho^{\prime 2}}\right)^{1 / 2}}{\bar{\rho}} \text { with } \gamma=1.4,
$$

and

$$
\overline{\rho^{\prime} u^{\prime}} \simeq 0.80\left(\overline{u^{\prime 2}}\right)^{1 / 2}\left(\overline{\rho^{\prime 2}}\right)^{1 / 2} .
$$

From Eqs. (16)-(18), we can obtain

$$
\overline{\frac{u^{\prime 2}}{U^{2}}} \simeq \frac{\overline{(\rho u)^{\prime 2}}}{\bar{\rho}^{2} U^{2}}\left[1+(\gamma-1)^{2} M_{a}^{4}+1.6(\gamma-1) M_{a}^{2}\right]^{-1} \text {. }
$$

From the assumption of constant static pressure and constant stagnation temperature across the boundary layer we have

$$
\frac{\bar{\rho}}{\rho_{\mathrm{ext}}} \simeq \frac{\operatorname{Re}^{2}}{\operatorname{Re}_{\mathrm{ext}}^{2}} \frac{M a_{\mathrm{ext}}^{2}}{M a^{2}} \text { and } \frac{\bar{U}}{U_{\mathrm{ext}}} \simeq \frac{M a^{2}}{M a_{\mathrm{ext}}^{2}} \frac{\operatorname{Re}_{\mathrm{ext}}}{\operatorname{Re}} .
$$

We can now write the explicit expression for $\bar{\rho} \overline{u^{\prime 2}} / \tau_{*}$ as

$$
\frac{\bar{\rho} \overline{u^{\prime 2}}}{\tau_{*}}=\frac{\bar{\rho}}{\rho_{*}} \frac{\overline{u^{\prime 2}}}{u_{*}^{2}}=\frac{1}{\rho_{*} u_{*}^{2}} \frac{\bar{\rho}}{\rho_{\mathrm{ext}}} \frac{\overline{u^{\prime 2}}}{U^{2}} \frac{U^{2}}{U_{\mathrm{ext}}^{2}} \rho_{\mathrm{ext}} U_{\mathrm{ext}}^{2},
$$

and using Eqs. (20) and (21) and the data listed above for the boundary layer, we obtain

$$
\frac{\bar{\rho} \overline{u^{\prime 2}}}{\tau_{*}}=186 \frac{\overline{(\rho u)^{\prime 2}}}{\bar{\rho}^{2} U^{2}} \quad \text { at } y / \delta=0.64
$$

and

$$
\frac{\bar{\rho} \overline{u^{\prime 2}}}{\tau_{*}}=190 \frac{\overline{(\rho u)^{\prime 2}}}{\bar{\rho}^{2} U^{2}} \text { at } y / \delta=0.32 .
$$

The CVA results for $\bar{\rho} \overline{u^{\prime 2}} / \tau_{*}$ calculated with the above expressions are shown in Fig. 11 superimposing the results of other earlier research. A total of three data points are shown in Fig. 11 that were obtained with the present CVA tests. One point is obtained by solving the three simultaneous overheat $(0.30,0.45$, and 0.62$)$ equations at $y / \delta=0.64$. This value is shown to be 1.21 on the $y$ axis in the graph. The two other points shown in Fig. 11 for the $\bar{\rho} \overline{u^{\prime 2}} / \tau_{*}$ that were 
computed from the CVA results at the two boundary layer points $\dot{y} / \delta=0.64$ (at $a_{w}=0.62$ ) and $y / \delta=0.32$ (at $a_{w}$ $=0.60$ ) are 1.11 and 1.52 , respectively. They were computed using the following approximate relationship neglecting the total temperature effect:

$$
\frac{\overline{e^{\prime 2}}}{E^{2}}=\left[S_{\rho u}^{\mathrm{CVA}}\right]^{2} \frac{\overline{(\rho u)^{\prime 2}}}{\bar{\rho}^{2} U^{2}} .
$$

From Fig. 11, it is very clear that the CVA results agree quite well with several other experiments, indicating that the CVA with an in situ measured time constant and software corrected thermal lag is indeed a viable option for quantitative measurements.

\section{ACKNOWLEDGMENTS}

The authors are indebted to Dr. Jean-Paul Bonnet, Head of the LEA-CEAT, Poitiers (France) for permitting the use of their supersonic tunnel facility and Dr. Stéphane Barre, Dr. Gérard Dury, Marie Christine Guégan, Philippe Szeger, and Serge Sapin who helped them in running the facility and data acquisition systems.

\footnotetext{
${ }^{1}$ G. R. Sarma, U.S. Patent No. 5074147 (1991).

${ }^{2}$ J. T. Lachowicz, N. Chokani, and S. P. Wilkinson, AIAA J. 34, 2496 (1996).
}

${ }^{3}$ A. E. Blanchard, J. T. Lachowicz, and S. P. Wilkinson, AIAA J. 35, 23 (1997).

${ }^{4}$ G. P. Doggett, N. Chokani, and S. P. Wilkinson, AIAA 35th Aerospace Sciences Meeting and Exhibit, Reno, Nevada, January, 1997, AIAA Paper No. 97-0557.

${ }^{5}$ M. A. Kegerise and E. F. Spina, ASME 3rd International Symposium on Thermal Anemometry, San Diego, California, July, 1996. (ASME-FED, 239).

${ }^{6}$ M. A. Kegerise and E. F. Spina, AIAA Fluid Dynamic Conference, Snowmass, Colorado, July, 1997, AIAA Paper No. 97-1955.

${ }^{7}$ G. Comte-Bellot, in Handbook of Fluid Dynamics, edited by R. W. Johnson (CRC Boca Raton, FL, 1998), Chap. 34.

${ }^{8}$ T. Moes, G. R. Sarma, and S. M. Mangalam, NASA TM-4806 (1997).

${ }^{9}$ S. M. Mangalam, G. R. Sarma, R. A. Pfouts, T. S. Kwa, J. H. Casper, M. A. Wallace, and R. Nigon, Report TS9801, Tao Systems, 1998.

${ }^{10}$ G. R. Sarma, Rev. Sci. Instrum. 69, 2385 (1998).

${ }^{11}$ E. Arzoumanian and J. F. Debieve, L'Onde Electrique 70, 55 (1990).

${ }^{12} \mathrm{P}$. Freymuth, DLR FB 66-03 (1966).

${ }^{13}$ S. Kuppa, G. R. Sarma, and S. M. Mangalam, ASME Fluid Measurement Instrumentation Forum FED 61, 67 (1993).

${ }^{14}$ G. Dury, Ph.D. dissertation, University of Poitiers, 1997.

${ }^{15}$ A. J. Smits and J. P. Dussauge, Turbulent Shear Layer in Supersonic Flows (AIP, New York, 1996).

${ }^{16}$ A. L. Kistler, Phys Fluids 2, 290 (1959).

${ }^{17}$ L. S. G. Kovasznay, J. Aeronaut. Sci. 20, 657 (1953).

${ }^{18}$ A. J. Smits, K. Hayakawa, and K. C. Muck, Exp. Fluids 1, 83 (1983).

${ }^{19}$ J. Gaviglio, J. Mécanique Appliquée 2, 449 (1978).

${ }^{20}$ S. Barre, P. Dupont, and J. P. Dussauge, Eur. J. Mech. B/Fluids 11, 439 (1992). 